Pacific Journal of Mathematics

ENLARGEMENTS OF QUANTUM LOGIC S 


\title{
ENLARGEMENTS OF QUANTUM LOGICS
}

\author{
Mirko Navara, Pavel Ptáx and Vladimír Rogalewicz
}

Let $K$ be a quantum logic whose state space is nonvoid. Let $B$ be a Boolean algebra and let $C$ be a compact convex subset of a locally convex topological linear space. Then $K$ can be enlarged to a logic $L$ such that the centre of $L$ equals $B$ and the state space of $L$ equals $C$. (The result remains valid when we replace the word "logic" with "orthomodular lattice".)

1. Introduction and preliminaries. An orthomodular partially ordered set $L$ ("a quantum logic") naturally induces one algebraic and one measure-theoretic structure: The centre, $C(L)$, which is the set of all absolutely compatible elements in $L$, and the state space, $\mathscr{S}(L)$, which is the set of all "states" (= probability measures) on $L$. When we investigate the interplay of $C(L)$ and $\mathscr{S}(L)$, a natural question arises whether one can construct logics with given centres, state spaces and preassigned sublogics. (This question seems to be of certain significance also from the physical point of view if one wants to clarify the dependence, resp. independence, of the centre and the state space in the model. It should be noticed that, for instance, in the von Neumann algebra model, the state space determines the centre-see [1], [6], [13].)

Since centres of logics are exactly Boolean algebras (see e.g. [5]) and since state spaces of logics are (up to an affine homeomorphism) exactly compact convex subsets of locally convex topological linear spaces (see [11]), our question reads as follows: Given a Boolean algebra $B$ and a compact convex set $C$ of LCTLS, can every logic be enlarged to a logic $L$ with $C(L)=B$ and $\mathscr{S}(L)=C$ ? In this paper we answer the latter question in the affirmative (obviously under "conditio sine que non" that the state space of the initial logic is nonvoid). We need rather advanced construction techniques with logics in some places-apart from applying key results and methods of the papers [3], [4], [9], [10], [11] we have to develop a fairly nontrivial "pasting" technique. This effort is needed mainly for establishing the following interesting lemma: Every logic $K$ with $\mathscr{S}(K) \neq \varnothing$ can be enlarged to 
a logic $L$ with card $\mathscr{S}(L)=1$. It turns out that this very special case of our problem becomes in fact equivalent to the general case.

Let us start the investigation with reviewing basic definitions and facts.

DEFINITION 1.1. A (quantum) logic is a partially ordered set $L$ with a least and a greatest element, 0,1 , together with an operation $x \rightarrow x^{\prime}$ (an orthocomplementation) mapping $L$ to $L$, such that the following conditions are satisfied for any $a, b \in L$ (the symbols $\vee, \wedge$ mean the lattice operations induced by $\leq$ ):

(i) $\left(a^{\prime}\right)^{\prime}=a$,

(ii) $a \leq b$ implies $b^{\prime} \leq a^{\prime}$,

(iii) $a \vee a^{\prime}=1$,

(iv) if $a \leq b^{\prime}$ then $a \vee b$ exists in $L$,

(v) if $a \leq b$ then $b=a \vee\left(a^{\prime} \wedge b\right)$ (the orthomodular law).

Typical example of logics is a Boolean algebra or the lattice of all projections in a von Neumann algebra. Naturally, a logic need not be either distributive or a lattice. In what follows, let us reserve the letter $L$ for logics.

Definition 1.2. Let $a, b$ be in $L$. We call $a, b$ orthogonal (abbr. $a \perp b$ ) if $a \leq b^{\prime}$, and we call $a, b$ compatible if there are mutually orthogonal elements $x, y, z \in L$ such that $a=x \vee z, b=y \vee z$. The centre $C(L)$ of $L$ is the set of all absolutely compatible elements $(C(L)=\{c \in L \mid c$ is compatible to any $d \in L\})$.

Proposition 1.3 (see [5]). The centre $C(L)$ of a logic $L$ is a Boolean algebra (with the operations $\vee, \wedge, '$ inherited from $L$ ). Further, we have $C(L)=L$ if and only if $L$ is a Boolean algebra. (Thus, obviously, every Boolean algebra is a centre of a logic.)

Definition 1.4. Let $K, L$ be logics and let $f: K \rightarrow L$ be a mapping. Then $f$ is called a (logic) morphism if the following conditions hold true:

(i) $f(0)=0$,

(ii) $f\left(a^{\prime}\right)=f(a)^{\prime}$ for any $a \in K$,

(iii) $f(a \vee b)=f(a) \vee f(b)$ whenever $a, b \in K$ and $a \perp b$.

When $f$ is bijective and both $f$ and $f^{-1}$ are morphisms, then $f$ is said to be an isomorphism. When $f: K \rightarrow f(K)$ is an isomorphism then $f$ is called an embedding. In this case we call $K$ a sublogic of $L$ and $L$ an enlargement of $K$. (Since a sublogic $K$ of $L$ is intrinsically identical with $f(K)$, we shall sometimes identify $K$ with $f(K) \subset L$.) 
Let us now introduce measure-theoretic notions we shall need in the sequel.

Definition 1.5. A state on a logic $L$ is a mapping $s: L \rightarrow\langle 0,1\rangle$ such that

(i) $s(1)=1$,

(ii) if $a, b \in L$ and $a \perp b$ then $s(a \vee b)=s(a)+s(b)$.

Let us denote by $\mathscr{S}(L)$ the set of all states on $L$ (called "state space"). The set $\mathscr{S}(L)$ is naturally endowed with a topological and convex structure (as a subset of $\langle 0,1\rangle^{L}$ ). When understood this way, $\mathscr{S}(L)$ is obviously a compact convex set. In fact, we have the following result.

Proposition 1.6 (see [11]). State spaces are (up to affine homeomorphisms) exactly compact convex subsets in locally convex topological linear spaces.

In the constructions which follow later we shall frequently use the following corollary of Proposition 1.6. There are logics $S, R$ with card $\mathscr{S}(S)=0$ (i.e., $\mathscr{S}(S)=\varnothing$ ) and card $\mathscr{S}(R)=1$ (i.e., $\mathscr{S}(R)$ is a singleton). We call the former logics stateless and the latter logics rigid. (Obviously, a stateless logic cannot be Boolean. There are nonBoolean rigid logics-for instance, it suffices to put $R=S \times\{0,1\}$ for a stateless logic $S$, see [10]).

Let us finally recall a simple construction. Observe first that the set $\langle 0, a\rangle=\{x \in L \mid x \leq a\}$ (resp. $\langle b, 1\rangle=\{x \in L \mid x \geq b\}$ ) becomes a logic when considered with the ordering and the orthocomplementation naturally inherited from $L$ (see e.g. [7], [12]). Using this fact, we can easily prove the following proposition.

Proposition 1.7. (A straightforward generalization of [3], Theorem 3.4; we can also obtain a proof of this proposition as a by-product of the final part of the proof of Theorem 2.2 in the next paragraph): Let $K, L$ be logics. Suppose that, for an element $a \in K \cap L$, we have $K \cap L=\langle 0, a\rangle \cup\left\langle a^{\prime}, 1\right\rangle$, where $\langle 0, a\rangle$ (considered as an interval in $K$ ) is isomorphic to $\langle 0, a\rangle$ (considered as an interval in $L$ ). Then $M=K \cup L$ becomes a logic (called the $\left(a, a^{\prime}\right)$-pasting of $K$ and $L$ ) if endowed with the partial ordering $\leq$ such that $a \leq b$ in $M$ if and only if $a \leq b$ in $K$ or in $L$, and with the orthocomplementation' inherited from $K$ and $L$. Moreover, both $K$ and $L$ become sublogics of $M$. 
Particularly, if $a=0$ we call the latter construction the $(0,1)$-pasting of $K$ and $L$. We have the following result which will be frequently used.

Proposition 1.8. Let $L$ be a logic. Let $S$ (resp. $R$ ) be a stateless (resp. rigid) logic. Then the following statements hold true:

(i) If $M$ denotes the $(0,1)$-pasting of $L$ and $S$ then $M$ is stateless. Moreover, if card $L>2$ then $C(M)=\{0,1\}$;

(ii) If $M$ denotes the $(0,1)$-pasting of $L$ and $R$ then $\mathscr{S}(M)$ and $\mathscr{S}(L)$ are affinely homeomorphic.

Moreover, if card $L>2$ and $\operatorname{card} R>2$ then $C(M)=\{0,1\}$.

The proof of Proposition 1.8 is elementary.

2. Results. The first result says that we solve our problem as soon as we can embed arbitrary logics into rigid logics. (As always in what follows, the sign "=" means "Boolean isomorphic" when applied for two Boolean algebras, and "affinely homeomorphic" when applied for two state spaces.)

Theorem 2.1. Let $R, H$ be logics and let $B$ be a Boolean algebra. Let us suppose that $R$ be rigid. Then $R$ can be embedded into a logic $L$ such that $C(L)=B$ and $\mathscr{S}(L)=\mathscr{S}(H)$.

Proof. Denote by $M$ the $(0,1)$-pasting of $R$ and $H$. Denote further by $P$ the $(0,1)$-pasting of $M$ and a stateless logic $S$. Then $\mathscr{P}(M)=$ $\mathscr{S}(H)$ and $\mathscr{S}(P)=\varnothing$. We may suppose that $C(M)=C(P)=\{0,1\}$.

Let us assume that $B$ is a Boolean algebra of subsets of a set $X$. Choose a point $z \in X$. Let $L$ be the set of all functions $f: X \rightarrow P$ with the following three properties:

(i) the range of $f$ is finite,

(ii) the set $f^{-1}(b)=\{x \in X \mid f(x)=b\}$ belongs to $B$ for any $b \in P$, (iii) $f(z) \in M$.

Let us endow the set $L$ with the usual "pointwise" partial ordering and orthocomplementation inherited from $P$. (We put $f=g^{\prime}$, resp. $f \leq g$, if $f(x)=g(x)^{\prime}$, resp. $f(x) \leq g(x)$, for any $x \in X$.) A routine verification gives that $L$ is a logic. Obviously, the mapping $h: R \rightarrow L$ such that $(h(k))(x)=k(k \in R, x \in X)$ is an embedding. It remains to be shown that $C(L)=B$ and $\mathscr{S}(L)=\mathscr{S}(H)$.

To show that $C(L)=B$, let us observe that a function $f \in L$ belongs to $C(L)$ if and only if $f(x) \in\{0,1\}$ for any $x \in X$. Indeed, if it is 
not the case, we have $f(x)=b \in M-\{0,1\}$. Since $C(M)=\{0,1\}$, there is an element $c \in M$ which is not compatible with $b$. Take a function $g: X \rightarrow L$ such that $g(x)=c$ for $f(x)=b, g(x)=f(x)$ otherwise. Then $g \in L$ and $g$ is not compatible to $f$. We see that $C(L)$ are exactly the "characteristic functions" of the subsets of $X$ which belong to $B$. Thus, $C(L)=B$.

Let us now consider $\mathscr{P}(L)$. Let us first notice that if $Y$ is a subset of $X$ such that $Y \in B$ and $z \notin Y$, then the set $L_{Y}$ of all constant functions $h: Y \rightarrow P$ forms a stateless logic (isomorphic to $P$ ). Suppose now that $s \in \mathscr{S}(L)$. By the foregoing property, we easily see that if $f \in L$ such that $f(z)=0$ then $s(f)=0$. Thus, for any $f \in L$, the value of $s(f)$ depends only on $f(z)$. It follows that the mapping $\alpha: \mathscr{S}(M) \rightarrow$ $\mathscr{S}(L)$ defined by the equality $\alpha(t)(f)=t(f(z))(t \in \mathscr{S}(M))$ is an affine homeomorphism and therefore $\mathscr{S}(M)=\mathscr{S}(L)$. The proof of Theorem 2.1 is complete.

The question now remains whether an arbitrary logic admits an embedding into a rigid logic. A positive answer to this question is given in the following theorem. It is obvious that the combination of Theorems 2.1, 2.2 establishes our main result.

THEOREM 2.2. Let $K$ be a logic and let $s$ belong to $\mathscr{S}(K)$. Then there is a rigid logic $R$ and an embedding $e: K \rightarrow R$ such that, for the (only) state $\tilde{s} \in \mathscr{S}(R)$, we have $\tilde{s} e=s$.

Proof. We shall need a few lemmas. Since the arguments are fairly technical in some places, the reader acquainted with the papers [3], $[10],[11]$ will probably find himself in a more convenient situation.

LemMA 2.3. Suppose that $\mathrm{H}_{3}$ is a three-dimensional Hilbert space and suppose that $L\left(\mathrm{H}_{3}\right)$ is the logic of all projections in $\mathrm{H}_{3}$. Suppose further that $a$ is an atom in $L\left(H_{3}\right)$ (i.e., we suppose that a is a projection on a one-dimensional subspace). Then there is precisely one state $s \in$ $\mathscr{S}\left(L\left(H_{3}\right)\right)$ such that $s(a)=1$ and, moreover, for any real number $r \in\langle 0,1\rangle$, we can find an atom $a_{r} \in L\left(H_{3}\right)$ such that $s\left(a_{r}\right)=r$.

Proof. Lemma 2.3 immediately follows from the description of states on $L\left(\mathrm{H}_{3}\right)$ given by the Gleason theorem (see [2]).

LEMMA 2.4. Let a real number $r \in\langle 0,1\rangle$ be given. Then we can construct a rigid logic $R_{r}$ such that, for an atom $a_{r} \in R_{r}$ and the (only) state $s \in \mathscr{S}\left(R_{r}\right)$, we have $s\left(a_{r}\right)=r$. 
Proof. For $r=1$, the rigid logic $R_{1}$ (with the appropriate atom $a_{1}$ ) was constructed in [11], Lemma 2. If $r \neq 1$, then we can choose an atom $a \in L\left(H_{3}\right)$ and identify $a$ with $a_{1}\left(a_{1} \in R_{1}\right)$ and, dually, $a^{\prime}$ with $a_{1}^{\prime}$. We also identify the zeros and units of $L\left(H_{3}\right)$ and $R_{1}$. Then the (a, $\left.a^{\prime}\right)$-pasting of $L\left(H_{3}\right)$ and $R_{1}$ can be taken for $R_{r}$ (Lemma 2.3).

LEMMA 2.5. Let $K$ be a logic and let $s$ belong to $\mathscr{S}(K)$. Choose an element $a \in K$. Then there is a logic $L_{a}$ such that the following conditions are satisfied (for a logic $L$ the symbol $\langle 0, x\rangle_{L}$, resp. $\left\langle x^{\prime}, 1\right\rangle_{L}$, means the interval $\langle 0, x\rangle$, resp. $\left\langle x^{\prime}, 1\right\rangle$, considered in $\left.L\right)$ :

(i) $K \cap L_{a}=\langle 0, a\rangle_{K} \cup\left\langle a^{\prime}, 1\right\rangle_{K}=\langle 0, a\rangle_{L_{a}} \cup\left\langle a^{\prime}, 1\right\rangle_{L_{a}}$ and the partial ordering and orthocomplementation in $K$ and in $L_{a}$ coincide on $K \cap L_{a}$

(ii) for any $t \in \mathscr{S}\left(L_{a}\right)$ we have $t(a)=s(a)$,

(iii) if $t_{1}, t_{2} \in \mathscr{S}\left(L_{a}\right)$ and $t_{1}(k)=t_{2}(k)$ for any $k \in K \cap L_{a}$, then $t_{1}=t_{2}$

(iv) there exists a state $\bar{s} \in \mathscr{S}\left(L_{a}\right)$ such that $\bar{s}(k)=s(k)$ for any $k \in K \cap L_{a}$.

Proof. Put $r=s(a)$. By Lemma 2.4 we can find a rigid logic $R_{r}$ and an atom $a_{r} \in R_{r}$ such that $t\left(a_{r}\right)=r$ for the state $t \in \mathscr{S}\left(R_{r}\right)$. In the $\operatorname{logic} R_{r}$ we first fill the interval $\langle 0, a\rangle_{K}$ in $\left\langle 0, a_{r}\right\rangle_{R_{r}}$ and identify $a$ with $a_{r}$. We obtain a set $\tilde{R}_{r}$. Further, for all $b \in R_{r}$ with $b \perp a_{r}$ and all $c \in\langle 0, a\rangle_{K}$, we add the elements $b \vee c$ to $\tilde{R}_{r}$ and identify $b \vee a$ with $b \vee a_{r}$. We obtain a set $L_{a}$. We endow $L_{a}$ with the partial ordering equal to the transitive closure of the orderings in $R_{r}$ and $\langle 0, a\rangle_{K}$ and with the orthocomplementation defined as follows: If $b \in L_{a}-R_{r}$, then we can write $b=c \vee d$, where $c \in\left\langle 0, a_{r}^{\prime}\right\rangle_{R_{r}}$ and $d \in\langle 0, a\rangle_{K}$, and define $b^{\prime}=\left(c^{\prime} \wedge a_{r}^{\prime}\right) \vee\left(d^{\prime} \wedge a\right)$, and if $b \in R_{r}$, then $b^{\prime}$ is defined as the orthocomplement of $b$ in $R_{r}$. It can be easily checked that $L_{a}$ becomes a logic. (The above construction is quite intuitive and thus we allow ourselves to leave the details to the reader. A thorough investigation of the construction together with further generalizations can be found in [8].)

The intersection $K \cap L_{a}$ consists of all elements of $\langle 0, a\rangle_{K}$ and their complements. Every state $t \in \mathscr{S}(K)$ restricted to $\langle 0, a\rangle_{K}$ has an extension to a state on $L_{a}$ if and only if $t(a)=s(a)$. We infer that $L_{a}$ possesses all the properties required in Lemma 2.5.

LEMMA 2.6. Let $K$ be a logic. Let us call a subset $S$ of $K$ overlapping if $a \leq b^{\prime}$ for no pair $a, b \in S$. Let us denote by $\mathscr{S}$ the collection of all 
overlapping subsets of $K$ and let us order the set $\mathscr{S}$ by inclusion. Then there is a maximal element $T$ in $\mathscr{S}$ and, moreover, for any $a \in K$ we have $a \in T$ or $a^{\prime} \in T$.

Proof. The existence of a maximal set $T \in \mathscr{S}$ follows immediately from Zorn's lemma. Suppose that $\left\{b, b^{\prime}\right\} \cap T=\varnothing$ for some $b \in$ $K$. Since $T$ is maximal, there exist $c, d \in T$ such that $c \leq b^{\prime}$ and $d \leq\left(b^{\prime}\right)^{\prime}=b$. It follows that $d \leq b \leq c^{\prime}$ and therefore $d \leq c^{\prime}$-a contradiction. This completes the proof of Lemma 2.6.

Let us now return to the proof of Theorem 2.2. Let us first take a maximal overlapping subset of $K$, some $T$, and put $T^{\prime}=\left\{a \in K \mid a=b^{\prime}\right.$ for some $b \in T$ \}. For each $a \in T^{\prime}$ take a logic $L_{a}$ from Lemma 2.5. We may (and shall) assume that $\left(L_{a}-K\right) \cap\left(L_{b}-K\right)=\varnothing$ for $a \neq b$. Let us put $\mathscr{R}=\{K\} \cup\left\{L_{a} \mid a \in T^{\prime}\right\}$. Let $R=\bigcup_{P \in \mathscr{R}} P$. Define $\leq, '$ in $R$ as follows: We have $c \leq d$ (resp. $c=d^{\prime}$ ) if and only if there is $P \in \mathscr{R}$ such that $c \leq d$ in $P$ (resp. $c=d^{\prime}$ in $P$ ). We claim now that $R$ is a logic with the properties required in Theorem 2.2. We first show that $R$ is indeed a logic. This will be verified in the following four statements.

Statement 1. If $P, Q \in \mathscr{R}$ and $P \neq Q$ then $P \cap Q$ is a subset of $K$ which is closed under the formation of the least upper bounds (in $K$ ) of orthogonal elements. Moreover, with any $x \in P \cap Q$ the set $P \cap Q$ contains either the entire set $\langle 0, x\rangle_{K}$ or the entire set $\langle x, 1\rangle_{K}$.

Indeed, we have

$$
\begin{aligned}
L_{a} \cap L_{b} & =\left(L_{a} \cap K\right) \cap\left(L_{b} \cap K\right) \\
& =\left(\langle 0, a\rangle_{K} \cup\left\langle a^{\prime}, 1\right\rangle_{K}\right) \cap\left(\langle 0, b\rangle_{K} \cup\left\langle b^{\prime}, 1\right\rangle_{K}\right) .
\end{aligned}
$$

Since both $a, b$ belong to $T^{\prime}$, we have ensured that $a^{\prime}$ is not orthogonal to $b^{\prime}$ and therefore $\langle 0, a\rangle_{K} \cap\left\langle b^{\prime}, 1\right\rangle_{K}=\varnothing=\langle 0, b\rangle_{K} \cap\left\langle a^{\prime}, 1\right\rangle_{K}$. We obtain

$$
L_{a} \cap L_{b}=\left(\langle 0, a\rangle_{K} \cap\langle 0, b\rangle_{K}\right) \cup\left(\left\langle a^{\prime}, 1\right\rangle_{K} \cap\left\langle b^{\prime}, 1\right\rangle_{K}\right) .
$$

Also, $L_{a} \cap K=\langle 0, a\rangle_{K} \cup\left\langle a^{\prime}, 1\right\rangle_{K}$. Thus, using this expression for $P \cap Q$, we immediately have that $P \cap Q$ possesses the required properties.

Statement 2. The relation $\leq$ is transitive on $R$.

Indeed, if $b \leq c$ in $P$ and $c \leq d$ in $Q$ for $P, Q \in \mathscr{R}$, then $c \in P \cap Q \subset$ $K$. The set $P \cap Q$ contains either the entire $\langle 0, c\rangle_{K}$ or the entire $\langle c, 1\rangle_{K}$. Hence either $b$ or $d$ belongs to $P \cap Q$. Thus either $P$ or $Q$ contains all the elements $b, c, d$ and therefore we have obtained $b \leq d$ as desired. 
Statement 3. Let $V$ be a subset of $R$ such that for any pair $x, y \in V$ there is a logic belonging to the collection $\mathscr{R}$ which contains both $x$ and $y$. Then the entire $V$ is contained in a logic belonging to $\mathscr{R}$.

Indeed, if $V$ is not contained in $K$, then there is an element $x$ such that $x \in V-K$. Then there is exactly one logic $P \in \mathscr{R}$ containing $x$ and therefore we have $V \subset P$.

Statement 4. The set $R$ is closed under the formation of the least upper bounds of orthogonal pairs.

Indeed, let $b, c \in R$ be orthogonal in $R$. Then there is a logic $P \in \mathscr{R}$ which contains the triple $\{b, c, b \vee c\}$, where the l.u.b. is taken in $P$. If $d$ is an upper bound of $b, c$ in $R$ then $b, c, d$ are contained in a logic $Q \in \mathscr{R}$ and therefore $b \vee c$ (in $P$ ) belongs to $P \cap Q$. Hence $b \vee c$ (in $P$ ) is not greater than $d$ (with respect to the ordering in $Q$ ) and we have $b \vee c($ in $P$ ) $=b \vee c($ in $R$ ).

It is obvious that the foregoing statements verify that $R$ is a logic. Moreover, the natural inclusion mapping $e: K \rightarrow R$ embeds $K$ into $R$. Finally, let $\tilde{s} \in \mathscr{S}(R)$. Since $T \cup T^{\prime}=K$, we see that $\tilde{s} e=s$. By the construction, every state on $L_{a}$ was completely determined by its values on $\langle 0, a\rangle$ (Lemma 2.5). Therefore $R$ admits only one state and the proof of Theorem 2.2 is complete.

Let us now state our main result. In view of the characterization of the state spaces of logics [11], we can formulate it as follows. (Moreover, if we take the trouble in verifying the lattice stability of the constructions-and the authors did-we can also add the lattice version.)

THEOREM 2.3. Suppose that we are given a Boolean algebra $B$ and a compact convex set $C$ of LCTLS. Then every non-stateless logic $K$ (resp. every non-stateless orthomodular lattice $K$ ) can be embedded in a logic $L$ (resp. can be lattice-theoretically embedded in an orthomodular lattice $L$ ) such that $C(L)=B$ and $\mathscr{S}(L)=C$.

Acknowledgment. The authors are grateful to Professor J. D. M. Wright and Dr. L. Bunce for their interest in the present results and for inspiring suggestions. 


\section{REFERENCES}

[1] J. Binder, On the interplay of the centre and the state space in quantum logics, Rep. Math. Phys., 24 (1986), 73-77

[2] A. Gleason, Measures on closed subspaces of a Hilbert space, J. Math. Mech., 6 (1957), 428-442.

[3] R. J. Greechie, On the structure of orthomodular lattices satisfying the chain condition, J. Combinatorial Theory, 4 (1968), 210-218.

[4] - Orthomodular lattices admitting no states, J. Combinatorial Theory, 10 (1971), 119-132.

[5] S. P. Gudder, Stochastic Methods in Quantum Mechanics, North Holland, New York, 1979.

[6] R. V. Kadison, Transformation of states in operator theory and dynamics, Topology, 3 (1965), 177-198.

[7] G. Kalmbach, Orthomodular Lattices, Academic Press, 1983.

[8] M. Navara and V. Rogalewicz, Pasting of quantum logics, to appear.

[9] P. Pták, Logics with given centres and state spaces, Proc. Amer. Math. Soc., 88 (1983), 106-109.

[10] _ Exotic logics, to appear in Colloq. Math., 1987.

[11] F. W. Shultz, A characterization of state spaces of orthomodular lattices, J. Combinatorial Theory, (A) 17 (1974), 317-328.

[12] V. Varadarajan, Geometry of Quantum Theory, Vol. 1, Van Nostrand, Princeton, N.J., 1968.

[13] F. J. Yeadon, Finitely additive measures on projections in finite $W^{*}$-algebras, Bull. London Math. Soc., 16 (1984), 145-150.

Received June 10, 1987.

Dept, of Mathematics

Faculty of Electrical Eng.

Technical University of Prague

16627 Prague, Czechoslovakia 



\section{PACIFIC JOURNAL OF MATHEMATICS \\ EDITORS}

\author{
V. S. VARADARAJAN \\ (Managing Editor) \\ University of California \\ Los Angeles, CA 90024 \\ HERBERT CLEMENS \\ University of Utah \\ Salt Lake City, UT 84112 \\ R. FINN \\ Stanford University \\ Stanford, CA 94305
}

\author{
HERMANN FLASCHKA \\ University of Arizona \\ Tucson, AZ 85721
}

vAUGHAN F. R. JONES

University of California

Berkeley, CA 94720

STEVENKERCKH OFF

Stanford University

Stanford, CA 94305

\author{
ROBION KIRBY \\ University of California \\ Berkeley, CA 94720 \\ C. C. MOORE \\ University of California \\ Berkeley, CA 94720 \\ HAROLD T ARK
}

University of California, San Diego La Jolla, CA 92093

\section{ASSOCIATE EDITORS}
R. ARENS
E. F. BECKENBACH
B. H. NEUMANN
F. WOLF
K. YOSHIDA

(1906-1982)

\section{SUPPORTING INSTITUTIONS}

\author{
UNIVERSITY OF ARIZONA \\ UNIVERSITY OF BRITISH COLUMBIA \\ CALIFORNIA INSTITUTE OF TECHNOLOGY \\ UNIVERSITY OF CALIFORNIA \\ MONTANA STATE UNIVERSITY \\ UNIVERSITY OF NEVADA, RENO \\ NEW MEXICO STATE UNIVERSITY \\ OREGON STATE UNIVERSITY
}

\author{
UNIVERSITY OF OREGON \\ UNIVERSITY OF SOUTHERN CALIFORNIA \\ STANFORD UNIVERSITY \\ UNIVERSITY OF HAWAII \\ UNIVERSITY OF TOKYO \\ UNIVERSITY OF UTAH \\ WASHINGTON STATE UNIVERSITY \\ UNIVERSITY OF WASHINGTON
}

The Supporting Institutions listed above contribute to the cost of publication of this Journal, but they are not owners or publishers and have no responsibility for its content or policies.

Mathematical papers intended for publication in the Pacific Journal of Mathematics should be in typed form or offset-reproduced (not dittoed), double spaced with large margins. Please do not use built up fractions in the text of the manuscript. However, you may use them in the displayed equations. Underline Greek letters in red, German in green, and script in blue. The first paragraph must be capable of being used separately as a synopsis of the entire paper. In particular it should contain no bibliographic references. Please propose a heading for the odd numbered pages of less than 35 characters. Manuscripts, in triplicate, may be sent to any one of the editors. Please classify according to the scheme of Math. Reviews, Index to Vol. 39. Supply name and address of author to whom proofs should be sent. All other communications should be addressed to the managing editor, or Elaine Barth, University of California, Los Angeles, California 90024.

There are page-charges associated with articles appearing in the Pacific Journal of Mathematics. These charges are expected to be paid by the author's University, Government Agency or Company. If the author or authors do not have access to such Institutional support these charges are waived. Single authors will receive 50 free reprints; joint authors will receive a total of 100 free reprints. Additional copies may be obtained at cost in multiples of 50 .

The Pacific Journal of Mathematics is issued monthly as of January 1966. Regular subscription rate: $\$ 190.00$ a year (5 Vols., 10 issues). Special rate: $\$ 95.00$ a year to individual members of supporting institutions.

Subscriptions, orders for numbers issued in the last three calendar years, and changes of address should be sent to Pacific Journal of Mathematics, P.O. Box 969, Carmel Valley, CA 93924, U.S.A. Old back numbers obtainable from Kraus Periodicals Co., Route 100, Millwood, NY 10546.

The Pacific Journal of Mathematics at P.O. Box 969, Carmel Valley, CA 93924 (ISSN 0030-8730) publishes 5 volumes per year. Application to mail at Second-class postage rates is pending at Carmel Valley, California, and additional mailing offices. Postmaster: send address changes to Pacific Journal of Mathematics, P.O. Box 969, Carmel Valley, CA 93924.

\section{PUBLISHED BY PACIFIC JOURNAL OF MATHEMATICS, A NON-PROFIT CORPORATION} Copyright (C) 1988 by Pacific Journal of Mathematics 


\section{Pacific Journal of Mathematics}

Vol. 135, No. $2 \quad$ October, 1988

Waleed A. Al-Salam and Mourad Ismail, $q$-beta integrals and the

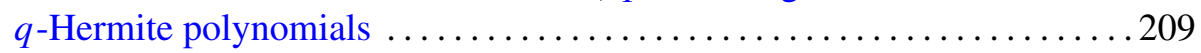

Johnny E. Brown, On the Ilieff-Sendov conjecture $\ldots \ldots \ldots \ldots \ldots \ldots \ldots 223$

Lawrence Jay Corwin and Frederick Paul Greenleaf, Spectrum and

multiplicities for restrictions of unitary representations in nilpotent Lie

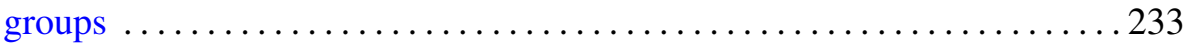

Robert Jay Daverman, 1-dimensional phenomena in cell-like mappings on 3-manifolds .......................................... 269

P. D. T. A. Elliott, A localized Erdős-Wintner theorem .............. 287

Richard John Gardner, Relative width measures and the plank problem . . . 299

F. Garibay, Peter Abraham Greenberg, L. Reséndis and Juan José

Rivaud, The geometry of sum-preserving permutations ............313

Shanyu Ji, Uniqueness problem without multiplicities in value distribution

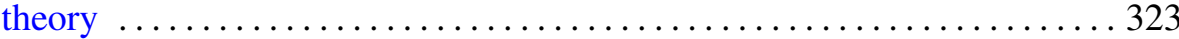

Igal Megory-Cohen, Finite-dimensional representation of classical

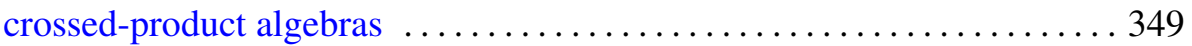

Mirko Navara, Pavel Pták and Vladimír Rogalewicz, Enlargements of

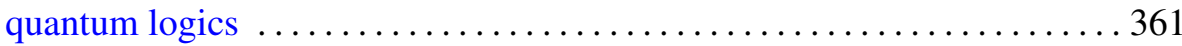

Claudio Nebbia, Amenability and Kunze-Stein property for groups acting

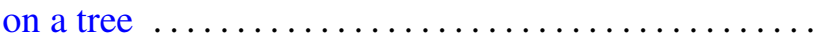

Chull Park and David Lee Skoug, A simple formula for conditional Wiener

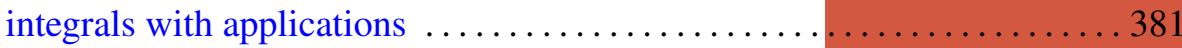

Ronald Scott Irving and Brad Shelton, Correction to: "Loewy series and simple projective modules in the category $\mathrm{O}_{s} " \ldots .$.

Robert Tijdeman and Lian Xiang Wang, Correction to: "Sums of products

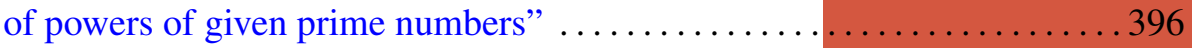

